\title{
Simulation of time-integrated dynamic speckle patterns in biomedical optics
}

\author{
Edward James*, Samuel Powell ${ }^{\dagger}$, Peter Munro* \\ *Department of Medical Physics \& Biomedical Engineering, University College London, London, WC1E 6BT, UK \\ ${ }^{\dagger}$ Faculty of Engineering, The University of Nottingham, University Park, Nottingham, NG7 2RD, UK
}

\begin{abstract}
The simulation of statistically accurate 2D time-integrated dynamic speckle patterns using a model that accounts for spatially varying sample properties (such as scatterer motion and decorrelation time) is yet to be presented in biomedical optics. We propose a solution to this important problem based on the Karhunen-Loève expansion of the field autocorrelation function, and apply our method to the formalisms of both laser speckle contrast imaging and diffuse correlation spectroscopy.
\end{abstract}

\section{Index Terms}

laser speckle, speckle imaging, speckle simulation, blood flow, biomedical optics

\section{INTRODUCTION}

The measurement of wide-field 2D time-integrated dynamic speckle patterns (2D-TIDSPs) for the purpose of blood flow measurement using inexpensive, low frame rate cameras, is an increasingly commonly performed task in the field of biomedical optics [1], [2]. Simulation techniques are a useful complement to experimental investigation with regard to understanding speckle phenomena and their practical application, as well as being a useful tool to evaluate data processing methods of speckle images, and assessing the feasibility of novel applications of laser speckle [3]. Despite this, an algorithm to simulate such images with statistical accuracy using a physics-based model that accounts for spatially varying sample properties is yet to be presented.

Two techniques have been described in the literature to model the evolution of a speckle pattern between two statistically independent fully-developed speckle patterns based on the field autocorrelation function, $g_{1}(\tau)$. The first of these is the copula method [4]; however, the solutions offered by this technique do not model static scatterers and are restricted to only a few prescribed forms of $g_{1}(\tau)$. The second technique, proposed by [3], allows for the generation of a series of fully developed speckle patterns with corresponding spatial variations in $g_{1}(\tau)$. This technique does not restrict the form of $g_{1}(\tau)$ that is used, and it is also able to model static scatterers. However, we have found that integrating over time in such an evolution does not simulate a 2D-TIDSP with statistically accurate speckle contrast, the property from which sample motion may be inferred.

Goodman employed the Karhunen-Loève expansion of the autocorrelation function to generate an exact solution for the probability density function (PDF) of a 2D-TIDSP for homogeneous media [5]. Here we extend this approach to the simulation of 2D-TIDSPs for heterogeneous media in biomedical optics. By doing so we build a robust forward model for 2D-TIDSPs that accounts for camera integration time, as well as spatially varying contributions from both dynamic and static scatterers.

\section{THEORY AND SIMULATIONS}

We wish to evaluate the intensity of a 2D-TIDSP as

$$
\mathbf{W}=\int_{0}^{T} \mathbf{U}(t) \mathbf{U}^{*}(t) d t
$$

where $\mathbf{U}(t)$, the complex valued field, is a random process that has autocorrelation function $g_{1}(\tau)$, where $\tau$ is the delay time $t_{2}-t_{1}$, and $g_{1}(\tau)$ measures the statistical similarity of $\mathbf{U}\left(t_{1}\right)$ and $\mathbf{U}\left(t_{2}\right)$. With a view to calculating 2D-TIDSP PDFs, Goodman showed that $\mathbf{W}$ can be expanded into an infinite series of statistically independent and uncorrelated fully developed speckle patterns [5]

$$
\mathbf{W}=\sum_{n=0}^{\infty}\left|\mathbf{b}_{n}\right|^{2}
$$

where $E\left[\left|\mathbf{b}_{n}\right|^{2}\right]=\lambda_{n}$, and $\lambda_{n}$ are the eigenvalues of $g_{1}(\tau)$. We therefore propose that a 2D-TIDSP may be simulated as the weighted sum of $N$ uncorrelated and statistically independent fully developed speckle patterns, each of which has a corresponding mean value of $E\left[\left|\mathbf{b}_{n}\right|^{2}\right]=\lambda_{n}$. We model a fully developed speckle pattern as $\mathbf{I}=|F(\mathbf{U})|^{2}$, where $F$ denotes the $2 \mathrm{D}$ Fourier transform and $\mathbf{U}=\exp (-i \boldsymbol{\Omega})$, where each $\boldsymbol{\Omega}$ is an independent instantiation of uniformly distributed random variables in a $2 \mathrm{D}$ matrix on the interval $(-\pi, \pi)[3]$. 
Here we use the example of $g_{1}(\tau)=\alpha\left|\exp \left(\frac{\tau}{\tau_{c}}\right)\right|+(1-\alpha)$, where $\tau_{c}$ is the decorrelation time of the sample and $\alpha$ is the fraction of the dynamic scattering component. This form of $g_{1}(\tau)$ can be used to model sample motion in both laser speckle contrast imaging (LSCI) and diffuse correlation spectroscopy (DCS) experiments [6], [7]. To evaluate $\lambda_{n}$ numerically, $g_{1}(\tau)$ is discretised into a 2D matrix over the camera exposure period, $T$. This is done by sampling the function $g_{1}\left(\left|t_{2}-t_{1}\right|\right)$ over an $N$ x $N$ matrix [Fig. 1 (a)] and calculating the eigenvalues of this square matrix [Fig. 1 (b)].
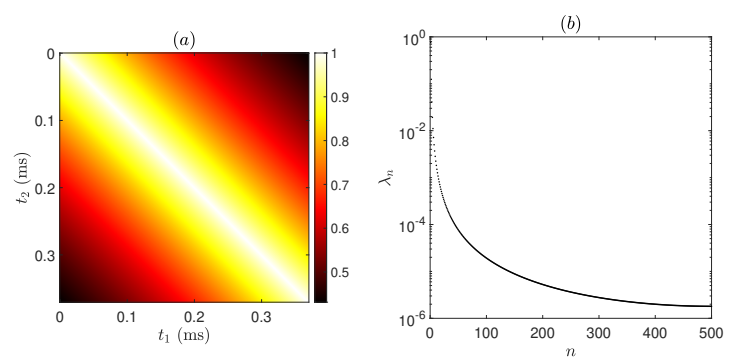

Fig. 1. (a) Sampling of $g_{1}\left(\left|t_{2}-t_{1}\right|\right)$ for $T=0.37 \mathrm{~ms}, \tau_{c}=0.37 \mathrm{~ms}, \alpha=0.9$ and $N=500$. (b) The $N$ eigenvalues, $\lambda_{n}$, of the square matrix (a).

An example of such a simulation, using the model parameters described in Fig. 1, is shown in Fig. 2(a). There is excellent agreement between the image histogram of this simulated speckle pattern and the expected PDF [Fig. 2(b)]. To simulate the spatially varying field of an in vivo sample, we adapted with permission the image of mouse ear vasculature from Fig. 5(a) of [8]. The image was segmented into five greyscale labels [Fig. 2(c)] and each label was assigned a value of $\tau_{c}$ in the range 0.1-1.0 ms. 30 2D-TIDSPs were simulated using values of $T=20 \mathrm{~ms}$ and $\alpha=1$, and a temporal speckle contrast image was computed from this stack of images. This is shown in Fig. 2(d), where the speckle contrast values correspond well with the spatial distribution of $\tau_{c}$ shown in Fig. 2(c).
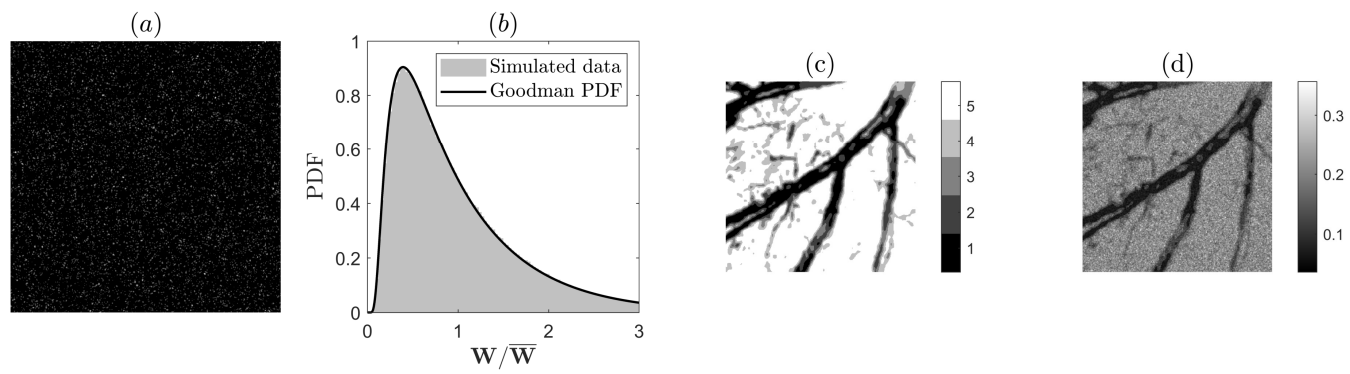

Fig. 2. (a) Simulation of a 2D-TIDSP using the $\lambda_{n}$ values shown in Fig. 1(b). (b) The image histogram of (a) and Goodman's expected exact PDF solution. (c) Vasculature image segmented into five labels. (d) Temporal speckle contrast image formed from 30 simulated 2D-TIDSPs.

\section{CONCLUSION}

We have presented a statistically robust and adaptable method to simulate 2D-TIDSPs in biomedical optics. This technique can readily be extended to account for different forms of homogeneous $g_{1}(\tau)$ (i.e., different types of motion, mixed motion models and alternative detection geometries), as well as spatial variations in $g_{1}(\tau)$ that result from a heterogeneous field. We expect the technique presented here to be a powerful and useful simulation tool for the biomedical optics community for the purposes of understanding the practical application of speckle phenomena, evaluating data processing methods of speckle images, and assessing the feasibility of novel applications of laser speckle.

\section{REFERENCES}

[1] K. Murali, A. K. Nandakumaran, T. Durduran, and H. M. Varma, "Recovery of the diffuse correlation spectroscopy data-type from speckle contrast measurements: towards low-cost, deep-tissue blood flow measurements," Biomedical Optics Express, vol. 10, no. 10, pp. 5395-5413, 2019.

[2] R. Bi, Y. Du, G. Singh, J.-H. Ho, S. Zhang, A. B. E. Attia, X. Li, and M. C. Olivo, "Fast pulsatile blood flow measurement in deep tissue through a multimode detection fiber," Journal of Biomedical Optics, vol. 25, no. 5, pp. 1-10, 2020.

[3] L. Song, Z. Zhou, X. Wang, X. Zhao, and D. S. Elson, "Simulation of speckle patterns with pre-defined correlation distributions," Biomedical Optics Express, vol. 7, no. 3, pp. 798-809, 2016.

[4] D. D. Duncan and S. J. Kirkpatrick, "The copula: a tool for simulating speckle dynamics," J. Opt. Soc. Am. A, vol. 25, no. 1, pp. 231-237, Jan 2008.

[5] J. Goodman, Statistical Optics, 2nd ed. Hoboken, New Jersey: Wiley, 2015.

[6] D. D. Postnov, J. Tang, S. E. Erdener, K. Kılıç, and D. A. Boas, "Dynamic light scattering imaging," Science Advances, vol. 6, no. 45, pp. 1-9, 2020.

[7] E. J. Sie, H. Chen, E.-F. Saung, R. Catoen, T. Tiecke, M. A. Chevillet, and F. Marsili, "High-sensitivity multispeckle diffuse correlation spectroscopy," Neurophotonics, vol. 7, no. 3, pp. $1-15,2020$.

[8] J. A. Guggenheim, J. Li, T. J. Allen, R. J. Colchester, S. Noimark, O. Ogunlade, I. P. Parkin, I. Papakonstantinou, A. E. Desjardins, E. Z. Zhang, and P. C. Beard, "Ultrasensitive plano-concave optical microresonators for ultrasound sensing," Nature Photonics, vol. 11, no. 11, pp. 714-719, 2017. 